\title{
Cuidado Humanizado en el Postoperatorio Inmediato de Pacientes Histerectomizadas
}

\section{Humanized Care in the Immediate Postoperative Period of Hysterectomized Patients \\ Cuidado Humanizado no Período Pós-Operatório Imediato de Pacientes Histerectomizados}

\author{
Yuneyda Camero Solórzano르, Isabel Meléndez Mogollón², Arelys \\ Álvarez Gonzalez ${ }^{3}$, Jenny Apuntes Guerrero ${ }^{4}$ \\ ${ }^{1}$ Especialista. Gerencia en Salud. Docente del Instituto Tecnológico Superior \\ Libertad (ITSL), Pichincha, Ecuador. Correo electrónico: \\ yuneydacameros@gmail.com \\ ${ }^{2}$ Licenciada en Enfermería. Departamento de Investigación y Docente del \\ Instituto Tecnológico Superior Libertad (ITSL), Pichincha, Ecuador. Correo \\ electrónico: isabelmelendez@gmail.com \\ ${ }^{3}$ Doctora en Ciencias Pedagógicas. Coordinadora Comisión de Creación, \\ Rediseño y Acreditación de Carreras del Instituto Tecnológico Superior \\ Libertad (ITSL). Correo electrónico: arelys.alvarez@itslibertad.edu.ec \\ ${ }^{4}$ Técnica Superior en Enfermería. Instituto Tecnológico Superior Libertad \\ (ITSL). Correo electrónico: ja_de78@yahoo.es
}

Cómo citar este artículo en edición digital: Camero, Y.B., Meléndez, I.C., Álvarez, A.R., \& Apuntes, Y.

E. (2019). Cuidado Humanizado en el Postoperatorio Inmediato de Pacientes Histerectomizadas.

Cultura de los Cuidados (Edición digital), 23 (54). Recuperado de

http://dx.doi.org/10.14198/cuid.2019.54.31

Correspondencia: Camero Solórzano Yuneyda Beatriz. Instituto Tecnológico Superior Libertad (ITSL). Av. 10 de Agosto N34-38 y Rumipamba Quito. Pichincha. Teléfonos: (02)-393-3850 - (02) 244-78-76

Correo electrónico de contacto: yuneydacameros@gmail.com ybcamero@itslibertad.edu.ec Recibido: 09/11/2018; Aceptado: 03/03/2019

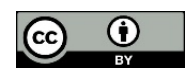

\begin{abstract}
Objective: To analyze the humanized care in the immediate postoperative period of hysterectomized patients, from $25^{\prime}$ s to 40 's, of the Olympus Clinic, during the period March-August 2017.
\end{abstract}

Method: The study is carried out under a quantitative approach, with a descriptive level, supported by a field investigation. The information was based on a 11 items questionnaire, which was applied to 18 nurses in order to obtain the required data.
Results: The application of humanized care is evidenced partially in the attention of biological needs in patients. It is noted that most of the respondents communicate effectively, however, a high level of indifference is manifested, on the part of nursing staff, in the pain attention as a basic need in the postoperative hysterectomy. Likewise, the absence of an instrument for evaluating user satisfaction is a weakness.

Conclusions: Humanized care is a complex but essential process during post-operative 
care in hysterectomies. Within the health institution addressed, it is developed in a fragmented manner, however, there is an opening in nursing professionals for the humanization of care.

Keywords: Humanized care, immediate postoperative, patients, hysterectomy.

\section{RESUMO}

Objetivo: Analisar o atendimento humanizado no pós-operatório imediato de pacientes histerectomizados, de 25 a 40 anos, da Clínica Olympus, durante o período de março a agosto de 2017.

Método: $\mathrm{O}$ estudo é realizado de forma quantitativa, com nível descritivo, apoiado em uma investigação de campo. As informações foram baseadas em um questionário de 11 itens, aplicado a 18 enfermeiros para obter os dados necessários.

Resultados: A aplicação do cuidado humanizado é evidenciada parcialmente na atenção das necessidades biológicas nos pacientes. Nota-se que a maioria dos entrevistados se comunica de forma eficaz, entretanto, manifesta-se um alto grau de indiferença, por parte da equipe de enfermagem, na atenção à dor como uma necessidade básica na histerectomia pósoperatória. Da mesma forma, a ausência de um instrumento para avaliar a satisfação do usuário é uma fraqueza.

Conclusões: $O$ cuidado humanizado é um processo complexo, mas essencial, durante os cuidados pós-operatórios nas histerectomias. Dentro da instituição de saúde abordada, desenvolve-se de forma fragmentada, no entanto, há uma abertura nos profissionais de enfermagem para a humanização do cuidado.

Palavras chave: Cuidado humanizado, pósoperatório imediato, pacientes, histerectomia.

\section{RESUMEN}

Objetivo: Analizar el cuidado humanizado en el postoperatorio inmediato de pacientes histerectomizadas, en edades de 25 a 40, de la Clínica Olympus, durante el período marzo-agosto 2017.

Método: El estudio se enmarca en un enfoque cuantitativo, con un nivel descriptivo, apoyado en una investigación de campo. La recolección de información se realizó a través de la técnica de la encuesta, con la aplicación de un cuestionario de 11 ítems a 18 enfermeras, para obtener los datos requeridos.

Resultados: La aplicación del cuidado humanizado se evidencia parcialmente en la atención de necesidades biológicas en las pacientes. La mayoría de los encuestados se comunican efectivamente, sin embargo, se manifiesta un alto nivel de indiferencia, por parte del personal de enfermería, en la atención del dolor como necesidad básica en el postoperatorio de histerectomía. Así también, es una debilidad la ausencia de un instrumento de evaluación de la satisfacción del usuario.

Conclusiones: El cuidado humanizado es un proceso complejo pero indispensable durante la asistencia de postoperatorio en histerectomías. Dentro de la institución de salud abordada, este cuidado se desarrolla de manera fragmentada, no obstante, existe una apertura en los profesionales de enfermería para la humanización de la atención.

Palabras clave: Cuidado humanizado, postoperatorio inmediato, pacientes, histerectomía.

\section{INTRODUCCIÓN}

La atención de enfermería es considerada un servicio que se otorga al usuario, en 
donde está inmersa la práctica del cuidado como parte de la calidad de prestación del sistema de ayuda. En el mismo orden de ideas, se afirma que la relación entre la enfermera y el paciente se enmarca en un fenómeno intersubjetivo y complejo, el cual garantiza un acceso al cuidado a la salud y un empoderamiento del paciente en sus propios autocuidados (Raile y Marriner, 2014).

En cuanto a la profesión de enfermería, se puede decir que es considerada una disciplina o conjunto de conocimientos que delimitan el ser y hacer de la profesión; esta profesión incluye elementos filosóficos, éticos y científicos que son empleados en la práctica. García y Téllez (2012), indican que la razón de ser de enfermería como profesión es el cuidado humano, es el paciente, por lo que se deben aplicar métodos, teorías, modelos o cualquier otro elemento conducente a cumplir con su objetivo.

De la misma manera, autores como Mastrapa y Gibert (2016), manifiestan que: "El cuidado como centro de atención de la enfermería, se describe también como la relación que se da entre la enfermera y el paciente con el fin de promover su salud, prevenir, diagnosticar, tratar y rehabilitar las enfermedades que puedan padecer". En tal sentido, se establecen relaciones con el paciente y familiares, y se favorece la relación terapéutica, además, se satisfacen las necesidades del usuario.

En cuanto a los aportes académicofilosóficos de las precursoras de la enfermería, Raile y Marriner, destacan que, Hildegard E. Peplau, describe la importancia de la relación entre la enfermera y el paciente como "un proceso interpersonal significativo y terapéutico, ya que se obliga a los pacientes a desarrollar respuestas destructivas o constructivas frente a: necesidad, frustración, conflicto y ansiedad" (Raile y Marriner, 2014). Es decir, que el profesional de enfermería desarrolla un proceso individual, basado en terapias en beneficio del paciente.

En el mismo orden de ideas, el término cuidar, como la acción pensada del profesional de enfermería, que es inherente a la humanidad, está basado en cuatro conceptos principales: enfermería, entorno, cuidado y salud, desde una perspectiva dinámica y cambiante, ya que el proceso salud-enfermedad no es estático (Téllez y García, 2012). Asimismo, Raile y Marriner (2014), al citar a Kerohuac, describen el cuidado a partir de tres paradigmas: 1) La categorización, donde el cuidado es visto de manera fraccionada como una suma de varias partes, de práctica institucionalizada, biologicista y con un enfoque médico; 2) La integración, que implica un cuidado integral, y toma en cuenta la persona, el entorno, la salud, el aspecto biológico, sicológico, social y espiritual; 3) La transformación del cuidado, que enfoca a la persona como un ser único. En este caso el cuidado es holístico y va más allá de lo cotidiano, convirtiéndose en un cuidado transformador.

En cuanto al tercer paradigma del cuidado, propuesto por Kerohuac, puede afirmarse que debe existir una creciente preocupación por parte de la enfermería para dispensar cuidados de salud creativos, cuyo centro de interés está en los cuidados de las personas, quienes, en la interacción continua con su entorno, viven sus propias experiencias de salud. En este caso, el cuidado se adecúa a las prioridades del paciente, y es dirigido a la consecución de su bienestar (Raile y Marriner, 2014). Cabe destacar que los modelos y teorías propuestos por R. R. Porse, M. Newman, M. Rogers y J. Watson quedan incluidos dentro de este paradigma. 
De lo anteriormente expuesto, se puede afirmar que el cuidado debe ser holístico. Sin embargo, desde el siglo XVIII, la atención del paciente ha estado centrada en los aspectos biomédicos y curativos; en este caso se reduce lo humano a lo biológico, pasando a ser el enfermo, una enfermedad (Suárez, 2012). En consecuencia, es necesario que las enfermeras como parte del equipo de salud, otorguen una atención centrada en el usuario, y que los cuidados humanizados se integren a la praxis para mejorar la calidad de la atención en los servicios de salud (Ariza, 2012).

Ciertamente, en la práctica diaria de la profesión, el personal de enfermería desempeña sus actividades como tareas repetitivas, ya sean propias o delegadas. Esto empobrece y hasta anula las acciones cuidadoras ante el equipo de salud (Poblete y Valenzuela, 2007), deforma el acto de cuidar, repercutiendo además en la imagen institucional y en la calidad de servicio que los centros de salud prestan. En base a lo anteriormente expuesto, la Organización Mundial de la Salud (2008), indica que a pesar de los avances tecnológicos que han venido desarrollándose a través del tiempo, existen fallas en la calidad y seguridad de la atención de los pacientes, con ocurrencia de eventos indeseados, perjudicando la representación social de las organizaciones de salud.

Por otra parte, los modelos y teorías de enfermería se fundamentan en una visión humanista del cuidado, lo que refiere que el cuidado debe ser para el profesional de enfermería su razón moral y no un procedimiento o una acción. El cuidar debe ser un proceso interconectado y de sensaciones compartidas entre la enfermera y paciente. En tal efecto, la teorizante J. Watson, pide unir la ciencia con las humanidades para que las enfermeras tengan un sólido fondo artístico liberal y entiendan otras culturas como requisito para utilizar la ciencia del cuidado y un marco mente-cuerpo-espiritual, lo que amplía la mente y mejora las habilidades de pensamiento y el crecimiento personal (Raile y Marriner, 2014).

En otras palabras, para que el cuidado sea humanizado, el personal de enfermería debe practicar una comunicación efectiva en todos los niveles en los cuales interactúa, utilizando algunos fundamentos tales como, el reconocer la situación y creencias de cada paciente, y explicar lo que se le va a realizar (Hermosilla, Mendoza y Contreras, 2016).

Dentro de este contexto, el Ilustre Colegio de Enfermería de Madrid, afirma que "La comunicación es un arte, una habilidad que debe ser dominada por el enfermero para hacerle frente a los pacientes y a sus familias, a los médicos, y al resto de profesionales sanitarios del equipo" (Ilustre Colegio de Enfermería de Madrid, 2015). Es por ello que el profesional de enfermería debe implementar técnicas de comunicación, reconocer al paciente por su nombre, dar confianza, ofrecer contacto visual $y$ tranquilidad a través del tacto.

Del mismo modo, es importante la aplicación del Proceso de Atención de Enfermería (PAE) en el manejo de pacientes en el postquirúrgico inmediato de pacientes histerectomizadas, ya que el PAE se presenta como una herramienta metodológica de trabajo, para identificar y satisfacer necesidades interferidas y resolver problemas de salud que afectan al ser humano, la familia y la comunidad. Es de interés destacar que, en el postoperatorio de la histerectomía, por constituir la extracción quirúrgica del útero, se requiere de cuidados individualizados que integren la asistencia de las necesidades biológicas, incluyendo aspectos endocrino metabólicos, y la 
atención psicológica que aborde el proceso de duelo que vive la paciente (Villamar, 2017).

Con respecto a las principales indicaciones para realizar una histerectomía, se expone que las principales causas son la "esterilización, los miomas uterinos gigantes, la displasia endometrial, el cáncer uterino, el dolor pélvico crónico, la endometriosis $y$ las masas anexiales benignas". Así mismo, menciona que, entre las complicaciones más frecuentes, asociadas tanto a la histerectomía vaginal como la abdominal, se pueden mencionar la fiebre y las infecciones del tracto urinario; otras menos frecuentes son la infección de la herida quirúrgica, las hemorragias, las lesiones vesicales y de uréteres (Villamar, 2017).

En relación al período post-operatorio inmediato, este abarca las primeras 24 horas después de la cirugía e incluye el tiempo en que el paciente permanece en la sala de recuperación post anestésica (SRPA) (Saager, et al., 2014). Este período se va a caracterizar, porque la paciente presenta alteraciones fisiológicas como inconsciencia, depresión cardiorrespiratoria, ausencia de sensaciones y del tono simpático, dolor e hipotermia, entre otros; es por ello por lo que se requiere de observación continua, control de signos vitales y de cuidados postanestésicos.

En concordancia con lo anterior, en la Unidad de Reanimación Post- Anestésica, el enfermo pasa relativamente poco tiempo, por lo que se deben valorar las necesidades de respiración y oxigenación, eliminación, actividad, bienestar, piel y mucosas, comunicación, relaciones y seguridad; y en base a estas necesidades, realizar los diagnósticos de enfermería y aplicar las intervenciones. Asimismo, es importante abordar el manejo del dolor, ya que puede provocar cambios en la función pulmonar, el aumento de la frecuencia respiratoria y reducción de la capacidad vital o reducción del volumen de reserva residual funcional, produciendo un aumento de las secreciones bronquiales, aparición de hipoxemia, neumonías y deterioro respiratorio (Ramírez, et al., 2014).

El siguiente cuidado en el postoperatorio es el control de infecciones, ya que existen factores de riesgo claramente relacionados con la morbilidad infecciosa, entre los que se destacan la falta de uso de antibiótico profiláctico, la cantidad de sangrado durante el procedimiento, y el cateterismo vesical prolongado posterior a la cirugía. Así mismo las complicaciones más frecuentes asociadas a la Histerectomía, son las infecciones de las vías urinarias, seguida de absceso de cúpula y hematoma de cúpula (Florián, 2015).

Para evitar la infección, se debe saber que las principales vías de contagio son la vía aérea y la infección cruzada desde heridas de otro paciente o desde otras heridas en distinta localización del mismo paciente mediante material contaminado. Por tal razón se puede interpretar que el cuidado de una herida es responsabilidad del personal de enfermería, así como su valoración y la aplicación del tratamiento adecuado (San Martín y Soto, 2014).

Con respecto al Cuidado Humanizado de Enfermería, se realiza un estudio para analizar el cuidado de enfermería en el postoperatorio inmediato de pacientes histerectomizadas, en edades de 25 a 40, de la Clínica Olympus en la ciudad de Quito. En este caso se puede mencionar que, en esta clínica, existe una alta frecuencia de actividad quirúrgica del área de ginecoobstetricia, en donde se efectúa la histerectomía como método anticonceptivo irreversible y procedimiento operatorio. 
Por otro lado, es importante mencionar que en esta institución se han reportado complicaciones en el postoperatorio inmediato de pacientes histerectomizadas, $\mathrm{y}$ fallas en la atención que debe proveer el personal de enfermería, por lo que se busca a partir del estudio de investigación, determinar estas deficiencias en la atención al paciente y evidenciar si se está realizando un cuidado humanizado de la salud a partir de la aplicación de normas y estrategias claras, que permitan prestar un servicio de calidad y eficiencia, garantizando además, que la atención sea continua, oportuna $y$ humana.

En este caso se evalúa el cuidado humanizado de enfermería a través del cumplimiento de algunos procedimientos en las pacientes histerectomizadas tales como: control de signos vitales, ejecución de cuidados personalizados en base a las necesidades del paciente, educación sobre el postoperatorio, comunicación sobre los procedimientos a realizar y contacto visual, respeto hacia la autonomía del paciente, acceso a sugerencias sobre los cuidados prestados, además de aplicación de protocolos de actuación.

Finalmente, el estudio permitirá que el profesional de enfermería identifique la forma de cómo en la actualidad se está aplicando el cuidado de enfermería, lo que dará paso a mejorar la calidad de la atención, a través de la implementación de cuidados continuos y oportunos, basados en el conocimiento científico, técnico $\mathrm{y}$ humanizado.

\section{MATERIALES Y MÉTODOS}

El tipo de investigación realizada es de campo, con fuentes obtenidas desde una realidad y nivel descriptivo, bajo un enfoque cuantitativo, utilizando la recolección de datos con base en la medición numérica y el análisis estadístico, analizando el comportamiento del fenómeno, en donde el cuidado de enfermería a nivel de pacientes post-histerectomizadas se otorga de forma repetitiva debido a la frecuencia de esta medida terapéutica. Se aplicaron los métodos inductivo-deductivo, analítico $\mathrm{y}$ sintético, a razón de interpretar la humanización de la asistencia y la relación enfermero-paciente, abordando el fenómeno dentro de su realidad dentro de las características institucionales (Hernández, Fernández, y Baptista, 2014).

La población y muestra fue de 18 personas, correspondientes a la totalidad de personal de enfermería que trabaja en la clínica Olympus. La recolección de datos se efectuó a través de un cuestionario constituido por 11 ítems de opción múltiple con selección única, referentes al cuidado humanizado otorgado a las pacientes histerectomizadas.

\section{RESULTADOS}

La administración del cuestionario aplicado a la población total de profesionales de enfermería que labora en la Clínica Olympus arrojó resultados diversos en relación al cuidado humanizado brindado a las pacientes histerectomizadas, tal como se presenta en la siguiente tabla. 
TABLA 1: Cuidado humanizado de enfermería en postoperatorio inmediato de pacientes histerectomizadas

\begin{tabular}{llcccccccccc}
\hline & & Siempre & Algunas veces & Nunca & Total \\
\cline { 2 - 7 } & F & $\%$ & F & $\%$ & F & $\%$ & F & $\%$ \\
\hline
\end{tabular}

$1 \quad$ Aplicación del control de signos vitales

como medio de interacción con el

paciente.

2 Ejecución de cuidados personalizados con base en el diagnóstico de enfermería y las necesidades del paciente.

3 Educación integral a las pacientes histerectomizadas y su grupo familiar

4 Comunicación con el paciente ofreciendo confianza, manteniendo contacto visual y lenguaje corporal.

5 Cumplimiento del consentimiento informado sobre la resolución quirúrgica y su tratamiento post operatorio.

6 Aplicación de la escucha empática

centrada en el paciente y sus impresiones.

0

0

$\begin{array}{llll}0 & 0 & 18 & 100\end{array}$

7 Manejo del dolor como parte de la atención prioritaria de la paciente histerectomizada.

8 Presencia de complicaciones

postoperatorias en las pacientes

histerectomizadas.

0

0

18

100

0

0

18

100

9 Aplicación de protocolo de atención a pacientes histerectomizadas basados en modelos de atención de enfermería.

10 Necesidad de formación del personal que realiza los cuidados en las pacientes histerectomizadas 
satisfacción del paciente en cuanto al

En estos resultados, se destaca de forma favorable que $94 \%$ de las encuestadas siempre realizan el control de signos vitales como medio de interacción con el paciente, $78 \%$ ejecutan cuidados personalizados en base al diagnóstico de enfermería y necesidades de la usuaria, y $72 \%$ mantienen comunicación con la histerectomizada, ofreciendo confianza, manteniendo contacto visual y lenguaje corporal.

Adicionalmente, se evidencia que $100 \%$ de las enfermeras escuchan empáticamente las impresiones de la paciente en relación con la experiencia de la intervención quirúrgica y sus necesidades y $100 \%$ de estas, respeta el principio de autonomía basado en un consentimiento informado y de libre actuación.

En cuanto al manejo del dolor, $67 \%$ de las encuestadas manifestó que siempre daban prioridad a esta manifestación clínica, mientras que $33 \%$ solo lo hacían algunas veces. No obstante, existen debilidades en la educación sanitaria impartida a las pacientes histerectomizadas, ya que $83 \%$ de los profesionales de enfermería nunca implementa educación integral a las pacientes y familiares sobre los aspectos relacionados con la intervención quirúrgica y su recuperación.

Por otro lado, $100 \%$ del personal de enfermería indicó que las pacientes algunas veces presentaban complicaciones postoperatorias, y que no contaban con protocolos de atención que estandarizaran y guiaran el cuidado en la atención de las pacientes histerectomizadas, por lo que tenían apertura para recibir formación sobre la atención adecuada que se les debe prestar a estas pacientes. Así mismo, 100\% de los profesionales encuestados expresó que nunca las pacientes valoraban el nivel de satisfacción sobre el cuidado de enfermería, y que había disposición de recibir sugerencias sobre la atención brindada para lograr humanizar el cuidado a través de acciones que representen un significado en el proceso de relación enfermero(a)paciente.

\section{DISCUSIÓN}

En el estudio se abordó la atención a una población vulnerable, como lo es la mujer en edad fértil, quien al someterse a una histerectomía transita por un período de riesgo y duelo, requiriendo una atención humanizada por parte del equipo de salud. Ante esta situación, el personal de enfermería debe contar con conocimientos que garanticen buenas prácticas y otorguen a estas pacientes apoyo, seguridad y confort.

Es de interés precisar que, en la Clínica Olympus de Quito, se evidencia que la mayoría de los profesionales de enfermería realizan procedimientos básicos tales como: control de signos vitales, ejecución de cuidados personalizados en base al diagnóstico de enfermería y necesidades del paciente; además, estos profesionales se 
comunican ofreciendo confianza y contacto visual, manteniendo un lenguaje corporal y escucha empática centrada en el usuario, dándole la oportunidad de manifestar sus malestares e impresiones, en concordancia con el modelo teórico de J. Watson. Este modelo conecta la experiencia y la percepción en la ejecución de prácticas habituales de cuidado, destacando el impacto que esto genera en el paciente, generando, a su vez, una sensación de seguridad (Raile y Marriner, 2014).

Sin embargo, existe un porcentaje importante de profesionales de enfermería que cumple parcialmente con algunos procedimientos, lo que afecta la humanización del cuidado, haciendo de esta una relación mecánica y biologicista, además de dar apertura a la aparición de complicaciones post operatorias y la aplicación de procedimientos de enfermería de manera empírica con un abordaje del cuidado poco interactivo. Estos aspectos se evidencian a través de las elevadas cifras de morbilidad y el alto número de reingresos en las áreas de ginecología, siendo una de las unidades más concurridas del espacio hospitalario (García, 2016). Es de interés reconocer que la histerectomía es una de las cirugías más practicadas en el mundo y genera fuertes reacciones emocionales con un reconocido efecto negativo sobre la recuperación posquirúrgica. Por esto se puede sostener, con base en toda la información buscada y su evidencia, que es imprescindible ofrecer alternativas quirúrgicas para todas las pacientes sometidas a procedimientos ginecológicos, ya que la cirugía de menor invasión y más conservadora traerá consigo menores problemas o complicaciones postoperatorias.

$\mathrm{Al}$ revisar la literatura científica con el fin de describir la histerectomía, sus diversas vías de abordaje y las actuaciones a realizar por parte del personal de enfermería para la elaboración de un plan de cuidados, es posible concluir que, para conseguir una mayor calidad asistencial y mejor adaptación física y psicológica en la mujer histerectomizada, es necesaria una preparación ajustada a las necesidades de cada paciente y sobre las consecuencias del tipo de cirugía, en la que la enfermería tiene un papel fundamental en la orientación, apoyo emocional y cuidados al alta (Franco, 2017). En base a lo anteriormente expuesto, se puede afirmar que la recuperación exitosa postquirúrgica de pacientes histerectomizadas y la disminución de sus complicaciones, dependen en gran parte de las orientaciones que estas pacientes y su grupo familiar reciban del profesional de enfermería sobre los cuidados de salud, a fin de facilitar el empoderamiento del autocuidado domiciliario. No obstante, esto no ocurre con los profesionales de enfermería que trabajan en la clínica Olympus, ya que en la mayoría de los casos la educación sanitaria es deficiente, en contradicción al fomento de buenas prácticas de salud, lo que produce aumento del riesgo en el postoperatorio inmediato de estas pacientes.

En tal sentido, Mastrapa y Gibert (2016), declaran que la relación enfermera-paciente se debe realizar con la finalidad de satisfacer las necesidades del usuario, promover la salud y favorecer la relación terapéutica con el paciente y familiares. Igualmente, la teoría de Watson sostiene que el cuidado humanizado representa una guía y apoyo a la práctica de enfermería, debido al beneficio de cultivar una conciencia de cuidados y al establecimiento de una relación terapéutica basada en valores humanísticos, con el fin de satisfacer las necesidades de los pacientes y mejorar su calidad de vida (Raile y Marriner, 
2014).

El manejo del dolor, como parte de la atención prioritaria de la paciente histerectomizada, se considera como un aspecto fundamental del cuidado de enfermería; sin embargo, un porcentaje importante del personal que trabaja en la clínica Olympus, algunas veces da importancia a este padecimiento, sin tomar en cuenta que el dolor es una de las necesidades humanas prioritarias según la jerarquización propuesta por Kalish (Téllez y García, 2012).

$\mathrm{Al}$ respecto, se observa que se pierde la relación terapéutica y la comunicación efectiva entre el cuidador y el enfermo y, más aún, se presenta este hecho como una implicación ético-legal. Ciertamente, la ética incluye acciones encaminadas a dar respuestas a las necesidades básicas del paciente, es pensar en el otro, anticipar sus penurias y darse cuenta de que el paciente existe; de ahí que la ética se contrapone a la indiferencia, evitando exponer al paciente a un sufrimiento previsible (Domusvi, 2011).

Por otra parte, es de vital importancia enfatizar que para la pronta recuperación de las pacientes histerectomizadas, se deben aplicar protocolos de atención de enfermería basados en modelos de atención, empleando conocimientos científicos que guíen las buenas prácticas de enfermería. En este caso, se evidencia que estos protocolos no son utilizados por el personal de enfermería de la clínica Olympus, en el ejercicio de sus funciones asistenciales. Es por ello que existe una alta probabilidad de que algunas de las complicaciones presentadas en estas pacientes, sean producto de un manejo terapéutico deficiente en las mismas.

En el mismo orden de ideas, García (2016), a partir de su estudio sobre procedimientos de enfermería en la atención a pacientes con heridas quirúrgicas, concluyó que las complicaciones postoperatorias fueron producto del deficiente manejo de heridas quirúrgicas por parte del personal de enfermería, entre las cuales se incluyen las histerectomías. En este caso el autor desarrolla una propuesta de elaboración de una Guía o Protocolo de Actuación de Enfermería para garantizar la calidad de atención de enfermería, el buen manejo de pacientes con heridas quirúrgicas, $\mathrm{y}$ disminuir sus complicaciones en las diversas instituciones de salud.

Cabe destacar que, Echeverría y Francisco (2017), indican que para ofertar cuidados de calidad en el área quirúrgica, las acciones de enfermería deben estar encaminadas a garantizar atención integral, personalizada y libre de riesgo, por lo que, para asegurar la calidad del cuidado del enfermero, se deben implementar protocolos de actuación y la capacitación del personal, a través de los cuales, se optimizará el cuidado de enfermería, se ofrecerá mayor seguridad a los pacientes del área quirúrgica, y en consecuencia mejorará la satisfacción del usuario por la atención recibida.

De allí, la necesidad de estandarizar el cuidado y capacitar a las enfermeras de la clínica Olympus que realizan los cuidados en las pacientes histerectomizadas, a través de la aplicación de protocolos que guíen una práctica de enfermería segura y adecuada al requerimiento de necesidades de las pacientes. Así mismo, es preciso que la totalidad del personal aplique un cuidado humanizado y transpersonal, integrando preceptos teóricos a razón de garantizar la pronta recuperación de las pacientes.

De igual manera, Montenegro (2013), concluyó que los cuidados de enfermería en el perioperatorio de histerectomía debe ser humanizado, ya que es el personal de enfermería quien mantiene mayor contacto con las pacientes, por lo que debe lograr 


\section{Cultura de los Cuidados}

interacción recíproca y empática, favoreciendo un clima de confianza durante el proceso de cuidado perioperatorio de las histerectomizadas.

En relación a lo anterior, surge la práctica de enfermería durante el cuidado postoperatorio a las pacientes histerectomizadas, centrada en una concepción holística, humana y con inclusión de la esfera afectiva, lo cual le otorga una nueva dimensión al cuidado, en donde la paciente logrará su recuperación a través de la relación intersubjetiva con el cuidador.

Esto consolida la teoría de hacer posible y transformar la atención de enfermería a través de una relación horizontal con el paciente, activo y partícipe de sus cuidados, asistido integralmente a partir del enfoque biopsicosocial determinado por la OMS, tal como se representa en la figura 1 , en donde se visibiliza el vínculo humanizado del enfermero(a) y la paciente.

\section{FIGURA 1: Relación humanizada con enfermero(a)-paciente}

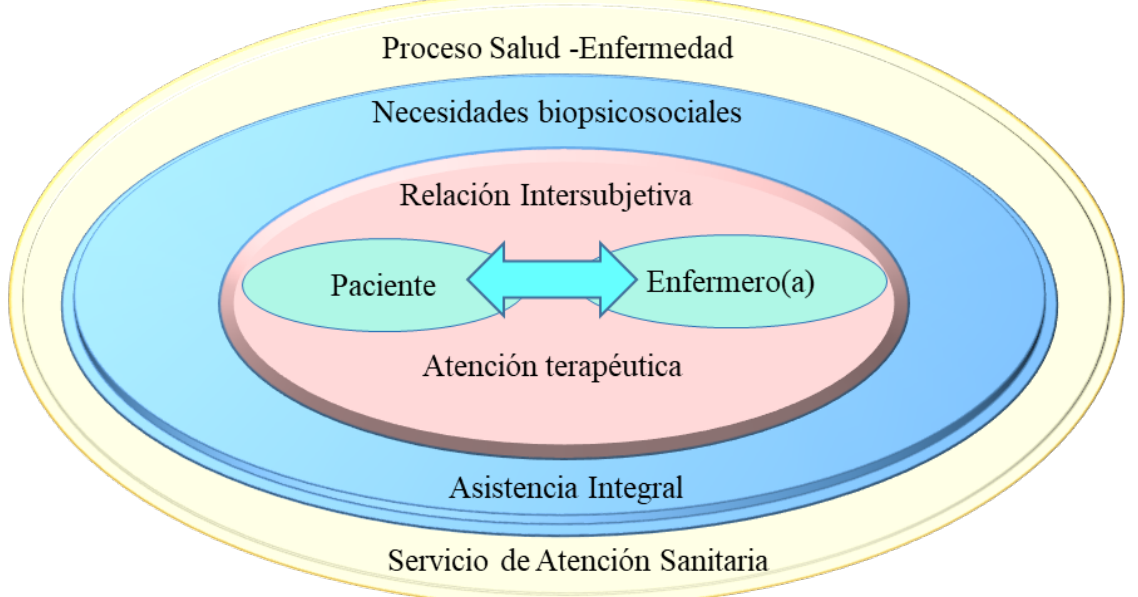

FuENTE: Camero, Meléndez, Álvarez y Apuntes (2018)

No obstante, las limitaciones presentes en la Clínica Olympus, invisibilizan a la realidad del paciente, ya que no existen instrumentos que evalúen la satisfacción en cuanto al cuidado de enfermería, lo que genera dificultades para evaluar la calidad de los cuidados. Asimismo, existe dificultad en la identificación de potencialidades, y las deficiencias en dichos cuidados, puesto que no hay evidencias que sustenten la reorganización de las actividades asistenciales, gerenciales y de enseñanza. 
FIGURA 2: Relación de los cuidados humanizados por el personal de enfermería

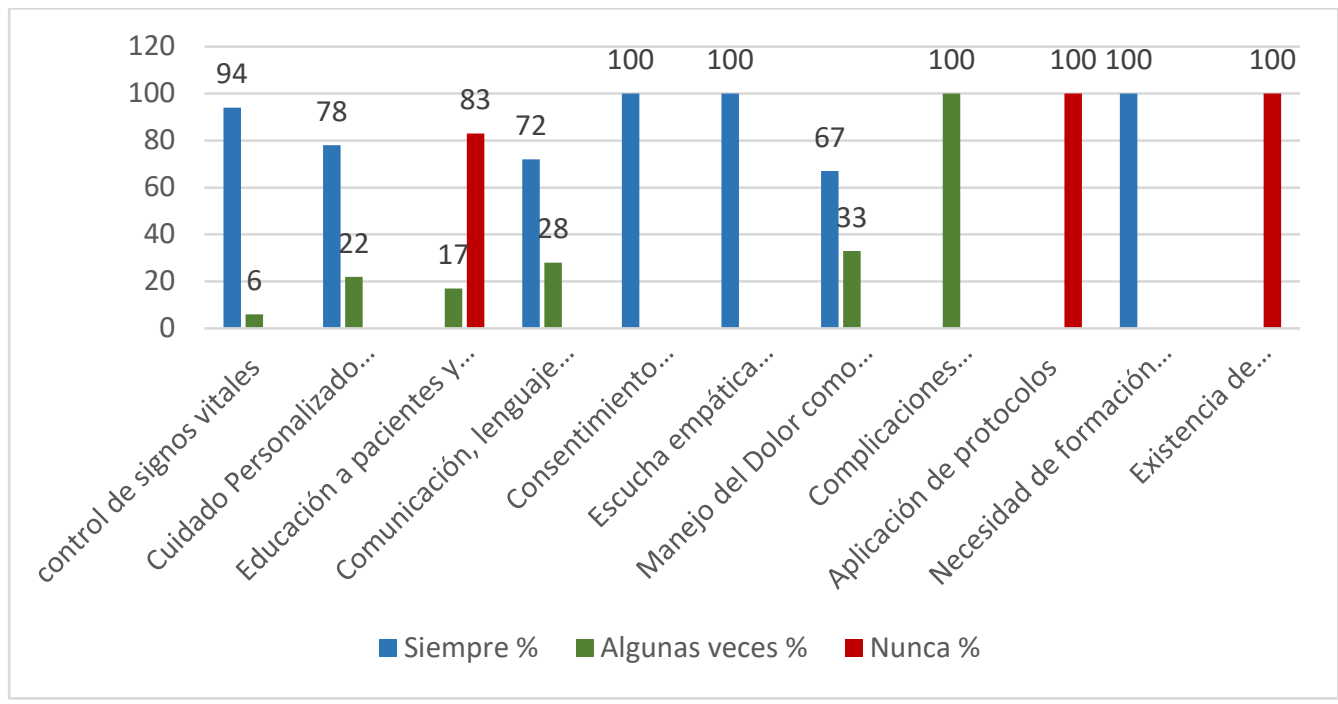

FuENTE: Camero, Meléndez, Álvarez y Apuntes (2018)

Tal como se presenta dentro de la figura 2, la relación de los cuidados tiene una tendencia con variaciones en elementos que fundamentan la humanización de los mismos, como lo es la comunicación, la aplicación de protocolos y la valoración de satisfacción.

\section{CONCLUSIONES}

El cuidado humanizado dirigido a las pacientes histerectomizadas, está constituido por la incorporación de los siguientes aspectos dentro del proceso de atención de enfermería: interacción con el paciente, cuidados personalizados, educación integral, comunicación, escucha empática, respeto a la autonomía, manejo del dolor y satisfacción del paciente. Estos elementos deben estar integrados en la prestación de cuidados, sin embargo, se aplican fragmentados en la atención de enfermería dentro de la Clínica Olympus, imposibilitando el desarrollo del cuidado humanizado.

Es importante mencionar, que existe un punto de partida dentro de todas las instituciones de salud del Ecuador, para iniciar un cambio en la cultura de los cuidados y la adopción de un modelo filosófico que sustente el quehacer de enfermería. Esto puede desarrollarse a partir de la apertura y receptividad propia que caracteriza al personal que ejerce el cuidado. En el caso del postoperatorio en histerectomía se recomienda la aplicación de estrategias en todos los niveles de gestión, entre las cuales se pueden mencionar los programas de educación continua, diseño de protocolos estandarizados, potenciación 
de los sistemas de comunicación institucional y evaluación del cuidado centrado en la satisfacción del paciente.

\section{AGRADECIMIENTO}

La culminación de cada etapa en la vida conlleva a reflexiones de las vivencias sentidas; es por ello que a partir de este pequeño espacio queremos agradecer tanto al personal que labora en la Clínica Olympus como al que trabaja Instituto Tecnológico Superior Libertad, a nuestros familiares, amigos y colegas de trabajo por la fe, confianza y solidaridad.

\section{BIBLIOGRAFÍA}

- Ariza, C. (Enero/Marzo de 2012). Soluciones de humanización en salud en la práctica diaria. Enfermaría Universitaria, 9 (1). Recuperado el 15 de 06 de 2018, de http://www.scielo.org.mx/scielo.php?script=sci artte xt\&pid=S1665-70632012000100006.

- Domusvi. (2011). Guía Práctica Ética Para Profesionales de la Salud. (SARcuavitae, Ed.) Recuperado de http://www.domusvi.es/wpcontent/uploads/2014/07/Guia_practica_etica_para_ profesionales de la salud.pdf.

- Echeverria, M., \& Francisco, J. (2017). Calidad del cuidado enfermero en un centro quirúrgico: Experiencia en un hospital de Ibarra, Ecuador. Enfermería Investiga, 2(4), 132-136.

- Florián, R. (2015). Factores Asociados a Infecciones Posoperatoriasen Histerectomía Vaginal en el Hospital Nacional Arzobispo Loayza. Recuperado file:///C:/Users/Docente\%201/Documents/ARTICUL OS/florian_rf.pdf.

- Franco, P. P. (2017). Histerectomía: tipos de abordaje y cuidados de enfermería. Trabajo fin de grado. Salamanca: Universidad de Salamanca. Recuperado de https://gredos.usal.es/jspui/handle/10366/133266.

- García Moreno, G. A. (2016). Procedimientos de enfermería en la atención a pacientes con heridas quirúrgicas y las complicaciones en el Hospital Enrique Garcés. Recuperado de http://dspace.uniandes.edu.ec/bitstream/123456789/3 121/1/TUAMEQ001-2016.pdf.

- Hermosilla, A., Mendoza, R., \& Contreras, S. (2016).
Instrumento para valoración del cuidado humanizado brindado por profesionales de enfermería a personas hospitalizadas. Index Enfermería, 25(4), 273-277.

- Hernández S, R., Fernández, C., \& Baptista, M. (2014). Metodología de la Investigación. 6ta edición (5ta ed.). Mexico: McGraw Hill.

- Ilustre Colegio de Enfermería de Madrid. (2015). Comunicación efectiva enfermera-paciente: Una estrategia más en el cuidado. Recuperado de https:/www.codem.es/noticias/comunicacionefectiva-enfermera-paciente-una-estrategia-mas-encuidado.

- Mastrapa, Y., \& Gibert, M. (2016). Relación enfermera-paciente: una perspectiva desde las teorías de las relaciones interpersonales. Revista Cubana de Enfermería, 32(4). Recuperado de http://www.revenfermeria.sld.cu/index.php/enf/arti cle/view/976/215.

- Montenegro Castañeda, I.K (2014). Cuidado enfermero en el perioperatorio de histerectomía, Hospital Luis Heysen Incháustegui-Chiclayo. Tesis de Grado USAT. Repositorio de Tesis USAT Recuperado de http://tesis.usat.edu.pe/handle/usat/431.

- Organizacion Mundial de la Salud. (2008). World Health Organization. Recuperado de http://www.who.int/patientsafety/information_centr e/documents/ps research brochure es.pdf.

- Poblete, T., \& Valenzuela, S. (Febrero de 2007). Cuidado humanizado: un desafío para las enfermeras en los servicios hospitalarios. Acta Paul Enferm 20(4)499-503.

- Raile, A., \& Marriner-Tomey, A. (2014). Modelos y Teorías de Enfermería. Madrid: Elsevier.

- Ramírez, C., Sánchez, L., González, D., Pérez, M., Martinez, M., Prado, J., \& Contreras, N. (2014). Evaluación del dolor en pacientes operadas de histerectomía abdominal. Rev Invest Med Sur Mex, 21(4), 156-160.

- Saager, L., Hesler, B., You, J., Turan, A., Mascha, E., Sessler, D., \& Kurz, A. (2014). Intraoperative transitions of anesthesia care and postoperative adverse outcomes. PubMed 121(4):695-706. doi: 10.1097/ALN.0000000000000401.

- San Martín, A., \& Soto, M. (2014). Cura de heridas quirúrgicas. Protocolo de actuación. Memoria de trabajo de fin de grado en Enfermería. Pamplona: Universidad de Navarra. Recuperado de http://academicae.unavarra.es/bitstream/handle/2454/11280/AguedaS anMartinLoyola.pdf?sequence=1.

- Suárez, M. (2012). Medicina centrada en el paciente. Revista Médica La Paz, 18(1), 67-72.

- Téllez, S., \& García, M. (2012). Modelos de Cuidados en Enfermería. NANDA, NIC, NOC. (1ra ed.). (H. G. México, Ed.) México D.F.: McGraw Hill.

- Villamar, K. (03 de Febrero de 2017). Proceso de 


\section{Cultura de los Cuidados}

Atención de Enfermería en Complicaciones Quirúrgicas de Histerectomías, sus principales causas y consecuencias. UTMACH. Unidad Académica de Ciencias Químicas y de la Salud. Recuperado de http://repositorio.utmachala.edu.ec/bitstream/48000/ 10255/1/VILLAMAR\%20ROGEL\%20KAREN\%20VA NESSA.pdf. 